\title{
HEPATITIS
}

\section{New biomarker identified for predicting spontaneous clearance of HCV infection}

Plasma levels of CXCL10 (also known as IFN- $\gamma$-inducible protein-10) are higher in patients with acute $\mathrm{HCV}$ infection who do not spontaneously clear the infection than in those who do, new data reveals.

Patients with acute HCV infection generally respond well to treatment; however, adverse effects are common and treatment is expensive. Tools to help predict which patients are likely to spontaneously clear the virus will therefore help clinicians to balance the benefits of early treatment against the potential for spontaneous viral clearance.

Jason Grebely (University of New South Wales, Australia) and colleagues analysed data from three cohorts of patients with acute HCV infection. Of 214 patients who had not received treatment, $14 \%$ achieved spontaneous viral clearance. Patients in whom spontaneous viral clearance did not occur had markedly higher plasma levels of CXCL10 at the time that acute HCV infection was detected than patients with spontaneous clearance. Furthermore, none of the patients with very high levels of CXCL10 ( $\geq 380 \mathrm{pg} / \mathrm{ml})$ achieved spontaneous clearance.

The authors conclude that CXCL10 could be a useful biomarker for determining which patients are unlikely to achieve spontaneous HCV clearance. "Early therapy may be considered in individuals with high levels of CXCL10, given the low likelihood of spontaneous clearance," says Grebely. The mechanism by which CXCL10 affects HCV clearance is unknown. However, the authors speculate that a high level of CXCL10 is a marker of an ineffective immune response rather than the cytokine interfering directly with viral clearance.

"Understanding who is less likely to spontaneously clear HCV infection provides useful clinical information for prioritizing patients for early antiviral therapy," says Grebely. The authors are

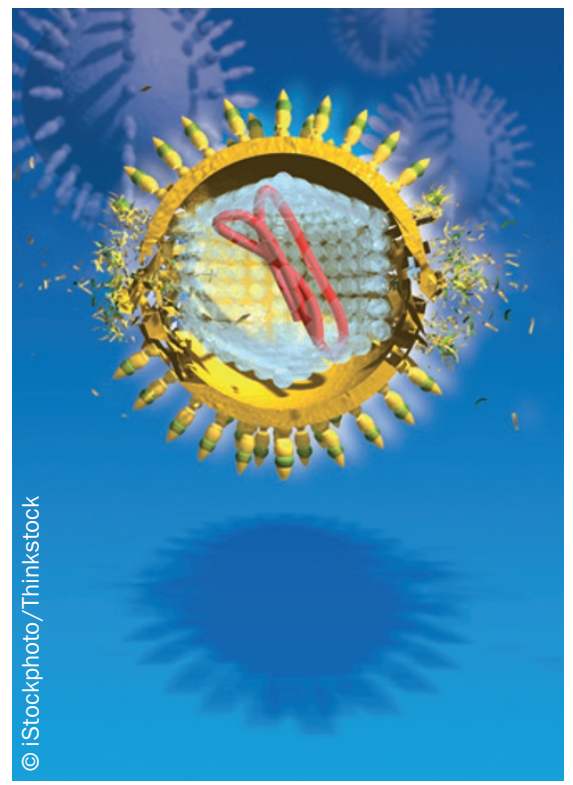

now exploring other cytokines and chemokines as potential biomarkers.

Claire Greenhill

Original article Grebely, J. et al. Plasma interferon- $\gamma$ inducible protein-10 (IP-10) levels during acute hepatitis C virus infection. Hepatology doi:10.1002/hep.26263 